\title{
Economic Analysis of Reproductive Management Strategies on US Commercial Dairy Farms
}

\author{
N. J. Olynk ${ }^{1}$ and C. A. Wolf \\ Department of Agricultural, Food, and Resource Economics, Michigan State University, East Lansing 48824
}

\begin{abstract}
Reproductive management has received increased attention in recent years as new technologies and programs have been developed to aid dairy managers in efficiently breeding cows and heifers. Efficient breeding of cows and heifers has become increasingly difficult as fertility has decreased because of a correlated negative response with milk yield. Dairy farm survey results were used to parameterize the economic analyses of reproductive management programs. Reproductive management program costs and the levels of reproductive performance achieved with such programs varied significantly among farms and were especially sensitive to on-farm labor costs. For example, visual heat detection requires more hours of labor per cow than use of an estrus synchronization program. Therefore, visual heat detection programs were relatively more sensitive to labor costs than synchronization programs. In addition, past reproductive performance was found to affect the determination of which reproductive program was optimal. Farms that had attained high levels of labor efficiency for visual heat detection in the past had less incentive to adopt a synchronization program than farms with less efficient visual heat detection.
\end{abstract}

Key words: reproduction, herd management, economics, synchronization

\section{INTRODUCTION}

The economic implications of reproductive management decisions are critical, given the link between dairy herd management and reproductive performance. Reproductive performance on the dairy farm affects profitability directly through the milk production per cow per day, number of replacements available, and voluntary and involuntary culling rates (Britt, 1985). Additionally, breeding costs and costs associated with veterinary care will affect the profitability of the dairy herd. Today, many reproductive technologies are

Received November 13, 2007.

Accepted June 5, 2008.

${ }^{1}$ Corresponding author: olynknic@msu.edu available for use on commercial dairy farms, with the costs and reproductive performance associated with these technologies varying considerably among farms. Reproductive management programs differ because of varying on-farm costs, housing and handling facilities, farm goals and values, and management styles.

Several survey-based studies in recent years have focused on dairy herd reproductive performance and management practices, providing a great deal of information. These overviews are useful for dairy producers, extension educators, researchers, and related farm service industries because they provide current information regarding management practices that have been adopted and used on commercial dairy farms. However, additional analysis is necessary to understand farm decisions relative to reproductive management programs and the resulting economics.

A recent analysis by Caraviello et al. (2006) examined survey results from 153 large US dairy herds in the Alta Genetics Advantage Progeny Testing Program in 2004. Caraviello et al. (2006) asked questions regarding general management, sire selection, reproductive management, inseminator training and technique, heat abatement, body condition scoring, facility design and grouping, nutrition, employee training and management, and animal health and biosecurity. Of the 103 herds whose managers completed the survey, the average herd size was 613 cows, and $87 \%$ of those herds used hormonal synchronization or timed AI (TAI) in their reproductive management programs. Caraviello et al. (2006) provided an in-depth reference of management practices being used on large commercial US dairy herds in 2004, which serves as a valuable resource for benchmarking or comparison purposes.

Meadows et al. (2005) also found decreasing marginal benefits arising from improved reproduction as reproductive performance improved. These decreasing marginal benefits to reproductive performance improvements explain why reproductive management strategies vary across farms. Those farms currently achieving high levels of reproductive performance had less incentive to initiate a potentially performanceenhancing change than did a farm with subpar current performance. 
Nebel and Jobst (1998) conducted a survey of bovine practitioners to evaluate the cost-effectiveness of systematic breeding programs. Using their survey results, they calculated estimated costs per pregnancy for Ovsynch and Targeted Breeding (Pharmacia-Upjohn, Kalamazoo, MI). The costs per pregnancy for drugs alone ranged from $\$ 5.75$ for Targeted Breeding with a $70 \%$ estrus detection rate at the least cost for drugs to $\$ 17.84$ for Ovsynch at the mean costs for drugs. Nebel and Jobst (1998) concluded that program costs must be weighed against reduced labor costs on estrus detection, and that cost-effectiveness must be calculated for each herd when assessing systematic breeding programs.

The objectives of this study were to provide economic insight into the reproductive management programs and technologies used on commercial dairy farms and to highlight the economic and management consequences of those programs. This analysis sought to build on prior reproductive management studies and dairy industry surveys by using survey data to inform the economic analysis of various reproductive management programs. Survey data were used to parameterize the economic analysis and inform the discussion regarding economic and management implications of reproductive management decisions. The expected net present values (NPV) of reproductive management programs were calculated to facilitate comparison.

\section{MATERIALS AND METHODS}

A survey was mailed to 1,000 dairy farms in Michigan, New York, Texas, Wisconsin, and Florida from August through December of 2006 to collect data regarding reproductive management and performance in 2005. Dairy farms receiving surveys were selected randomly from those permitted to sell grade A milk in the aforementioned states, thereby allowing a broad range of farms to participate in the survey. The number of farms receiving surveys in each state was selected proportionately to the total number of dairy farms in that state. A description of Ovsynch, Presynch with Ovsynch, Heatsynch, Cosynch, controlled internal drug-releasing intravaginal insert (CIDR) containing progesterone with $\mathrm{PGF}_{2 a}$, and the Targeted Breeding Protocol were provided as an appendix to the reproductive management survey for reference.

A total of 102 surveys were returned, for a $10.2 \%$ response rate. Only those respondents who were actively operating dairy farms in 2005 and who chose to participate in the survey were included in this analysis. Of the 102 respondents, only 60 were actively dairy farming during 2005 and were willing to participate in the survey, resulting in a total of 60 potential respondents for each question. Respondents who refused to participate, had exited dairying before 2005, or who operated related businesses such as a custom heifer raising operation were not included in the analysis. Consistent with Michigan State University research requirements when administering a survey, respondents were presented with the option of declining to answer individual questions or sections of the survey at their discretion, if they chose to participate at all.

The random selection of farms to receive the survey allowed an equal opportunity for selection, regardless of participation in various farm programs or membership in a particular cooperative. Although farms were randomly selected to receive surveys, given the relatively small response rate, the sample was not expected to be representative of the diverse population of US dairy farms. However, the survey itself was not the primary focus of this analysis. The survey data were used to parameterize the analysis of factors affecting the decisions of farms to use various reproductive management programs. The management and economic implications of various reproductive management programs were explored through sensitivity analysis.

Throughout the results, the "number of total responses" accompanies summary statistics, which indicates the total number of usable responses to a given question. Many questions allowed a respondent to check all answers that were applicable to the operation from a multiple-choice list, and such questions were analyzed by tabulating the total number of responses and then computing frequencies.

Budgets were developed in Excel (Microsoft, Seattle, WA) to determine farm-specific costs associated with achieving various levels of reproductive performance. The time required to administer injections is an important component of cost that is a function of the facilities used and skill level. Similarly, visual heat detection program costs vary depending on hourly labor costs and the efficiency with which heats are detected. Reproductive program costs were calculated on a percow basis to facilitate comparison across programs and herd sizes. Heat detection program costs were adjusted to obtain a per-cow basis by dividing the cost of heat detection for a group of cows by the number of cows in the group.

The synchronization protocol chosen for use in this example was Ovsynch because it was the most common synchronization program used among the surveyed operations (38\% of those respondents using synchronization used Ovsynch for their cows). Ovsynch consists of 1) an injection of $\mathrm{GnRH}, 2$ ) an injection of $\mathrm{PGF}_{2 \alpha} 7 \mathrm{~d}$ later, 3) a second $\mathrm{GnRH}$ injection $48 \mathrm{~h}$ later, and 4) timed breeding $24 \mathrm{~h}$ after the second GnRH injection (Pursley et al., 1995). To resynchronize cows not conceiving to the first AI, all cows were assumed to receive a GnRH 
injection at a nonpregnancy diagnosis (assumed to be $33 \mathrm{~d}$ post Ovsynch TAI), a $\mathrm{PGF}_{2 \alpha}$ injection $40 \mathrm{~d}$ after the initial Ovsynch TAI, and a second GnRH injection and TAI approximately $42 \mathrm{~d}$ after the initial Ovsynch TAI. For comparison, resynchronization beginning 26 d after Ovsynch TAI was assessed. Fricke et al. (2003) compared these resynchronization programs with other programs with altered timing to the beginning of resynchronization. The assumptions described here were used to create baseline farm scenarios.

To compare values across synchronization programs, the values must be assessed subject to achieving a specified level of reproductive success and discounted to a specific point in time. For instance, scenarios can be assessed in which cows are bred for a predetermined number of AI. In this analysis, scenarios were assessed in which cows were bred for $6 \mathrm{AI}$.

Whereas heat detection rate is the percentage of cows correctly detected in estrus in a $21-\mathrm{d}$ period, AI submission rate is the percentage detected in estrus and bred in that same period. The cumulative probability of pregnancy was defined as the sum of the probability of pregnancy from the first AI through the most recent AI. In calculating the number of services necessary to reach a cumulative probability of pregnancy, the conception rate (CR) was assumed to decrease as the AI number increased. The second and third or later AI were assumed to be 90.7 and $81.4 \%$ of the first AI CR, based on the results of Cassell (2001). The AI submission rate was held constant for each program.

Once the predetermined cutoff (cumulative probability of pregnancy of $90 \%$ or a specified number of AI) was achieved, the cow's value was included by incorporating her retention payoff (RPO). The RPO is the difference in total net returns from keeping the cow in the herd versus culling and replacing her immediately. The RPO is defined as the total additional profit expected from attempting to keep the cow until her optimal age compared with immediately removing her from the herd and replacing her (taking into account changes in involuntary culling); the greater the $\mathrm{RPO}$, the more valuable the animal, thus the larger the loss if the cow is culled at that time (Groenendaal et al., 2004). The RPO values used were obtained by using DairyVIP version 1.1 (De Vries, 2006). Input values used to obtain the RPO for pregnant and open cows were from De Vries (2006) unless otherwise specified below.

For each period, the RPO of a cow that conceived in that period was multiplied by the $\mathrm{CR}$ for that period and discounted. The probability that the cow remained open after the last breeding period was multiplied by the RPO of an open cow in the period in which the cutoff criterion was reached. The cost per breeding for calcu- lating the RPO was set at zero because breeding costs were accounted for in calculating the NPV of the breeding programs. The RPO values included in the model were greater than zero, and values that were negative were evaluated at zero. Feed and yardage of $\$ 60.80$ (assumed at $\$ 2 / \mathrm{d}$ for $30.4 \mathrm{~d}$ ) was charged in calculating the breeding program cost for each period that the cow remained open after her first AI. An annual discount rate of $9 \%$ was used for all calculations, as an entry for the RPO calculation as well as for calculating the NPV of the breeding programs (Wolf et al. 2002). Cows were assumed to be bred beginning in their third month in lactation and were bred once during every eligible period until the cutoff criterion was reached. Eligible periods for breeding differed based on the program in which cows were being bred. Cows bred with visual heat detection were assumed to have breeding periods of $21 \mathrm{~d}$. Cows on a synchronization program were assumed to begin resynchronization upon a nonpregnant diagnosis at either 26 or $33 \mathrm{~d}$ after TAI.

Heat detection program costs, assessed on a perperiod, per-cow basis, were calculated as follows:

$$
\begin{gathered}
\mathrm{PROG}_{\mathrm{HD}}=(\{[(\mathrm{TIME} \times \mathrm{OBS}) \times \mathrm{PER}] \\
\left.\left.\times \mathrm{LABOR}_{\mathrm{HD}}\right\} / \mathrm{COWS}\right)+\mathrm{AID}+(\mathrm{CI} \times \mathrm{SUBRATE}),
\end{gathered}
$$

where $\mathrm{PROG}_{\mathrm{HD}}$ is heat detection program costs per cow per period, TIME is heat detection minutes per observation for a group of cows, OBS is the number of times the group is observed per day, PER is the number of days in a single breeding period, $\mathrm{LABOR}_{\mathrm{HD}}$ is the cost of labor (in dollars per minute) to perform heat detection, COWS is the number of cows in the group, AID is the per-period cost per cow of the heat detection aid used, CI is the cost of an AI, and SUBRATE is the AI submission rate.

Synchronization program costs, assessed on a perperiod, per-cow basis, were calculated as follows:

$$
\begin{aligned}
& \text { PROG }_{\text {Synch }}=\left(\mathrm{P}_{\mathrm{GnRH}} \times \mathrm{X}_{\mathrm{GnRH}}\right)+\left(\mathrm{P}_{\mathrm{PGF} 2 \alpha} \times \mathrm{X}_{\mathrm{PGF} 2 \alpha}\right) \\
& +\left(\mathrm{MIN} \times \mathrm{INJ} \times \text { Labor }_{\mathrm{Inj}}\right)+(\mathrm{CI} \times \mathrm{SUBRATE}),
\end{aligned}
$$

where $\mathrm{PROG}_{\text {Synch }}$ is the synchronization program costs per cow per breeding period, $\mathrm{P}_{\mathrm{GnRH}}$ is the cost of $\mathrm{GnRH}$ per injection, $\mathrm{X}_{\mathrm{GnRH}}$ is the number of $\mathrm{GnRH}$ injections administered, $\mathrm{P}_{\mathrm{PGF} 2 \alpha}$ is the cost of $\mathrm{PGF}_{2 \alpha}$ per injection, $\mathrm{X}_{\mathrm{PGF} 2 \alpha}$ is the number of $\mathrm{PGF}_{2 \alpha}$ injections administered, MIN is the number of minutes to give a single injection, INJ is the total number of injections in the series, $\mathrm{LABOR}_{\text {Inj }}$ is the cost of labor (in dollars per minute) to give injections, $\mathrm{CI}$ is the cost of an AI, and SUBRATE is the $\mathrm{AI}$ submission rate. 
In calculating the program costs for both synchronization and visual heat detection programs, the cost of labor to perform the program was assessed as wage paid if the task was performed by paid labor or as opportunity cost of the labor if unpaid labor was used. Opportunity cost is the cost of having that labor participate in the reproductive management tasks rather than in the next best alternative activity. Because many herds, especially smaller herds, use unpaid family or managerial labor for their reproduction program, to assess the true costs associated with various reproductive management programs a charge for the opportunity cost of unpaid labor must be included. In many cases, such as when managers perform heat detection, the opportunity cost for unpaid labor was more expensive than for hired labor.

The following formula was used to calculate the expected NPV of each program, with $\mathrm{NCF}_{\mathrm{T}}$ for the last time period including the RPO values:

$$
\begin{gathered}
\mathrm{NCF}_{\mathrm{t}}=\left[\mathrm{CR}_{\mathrm{t}} \times \mathrm{RPOPREG}_{\mathrm{t}}-\left(1-\mathrm{PR}_{\text {cum }}\right)\right. \\
\left.\times \text { Feed }-\mathrm{PROG}_{\mathrm{j}}\right]
\end{gathered}
$$

for $t=0, \ldots, \mathrm{T}-1$, and

$$
\begin{gathered}
\mathrm{NCF}_{\mathrm{T}}=\left[\mathrm{CR}_{\mathrm{T}} \times \mathrm{RPOPREG}_{\mathrm{T}}-\left(1-\mathrm{PR}_{\text {cum }}\right)\right. \\
\left.\times \text { Feed }-\mathrm{PROG}_{\mathrm{j}}+\left(1-\mathrm{PR}_{\text {cum }}\right) \times \mathrm{RPOOPEN}_{\mathrm{T}}\right]
\end{gathered}
$$

for $t=\mathrm{T}$, where $\mathrm{NCF}_{\mathrm{t}}$ is the net cash flow in period $\mathrm{t}$; PROG is the program costs for either the synchronization or heat detection program per cow per period; $\mathrm{CR}_{\mathrm{t}}$ is the current $\mathrm{AI}$ CR; RPOPREG $\mathrm{t}_{\mathrm{t}}$ is the retention payoff in time $t$ for a pregnant cow; $\mathrm{PR}_{\text {cum }}$ is the cumulative probability of pregnancy, defined as the sum of probability of pregnancy from 0 through the current $\mathrm{AI}$, in time $\mathrm{t}$ [e.g., $\mathrm{PR}_{\text {cum }, 3}=\mathrm{CR}_{0}+\left(1-\mathrm{CR}_{0}\right) \times \mathrm{CR}_{1}+$ $\left.\left(1-\mathrm{CR}_{0}\right) \times\left(1-\mathrm{CR}_{1}\right) \times \mathrm{CR}_{2}\right]$; Feed is the per-period feed and yardage cost of a nonpregnant cow $(\$ 2.00 / \mathrm{d} \times$ days per period); $P_{R O G}$ is the cost of program $j$, where $j$ is the reproductive program used on the cow; RPOOPEN $\mathrm{N}_{\mathrm{T}}$ is the retention payoff in time $\mathrm{T}$ for an open cow; and $\mathrm{RPOPREG}_{\mathrm{T}}$ is the retention payoff in time $\mathrm{T}$ for a pregnant cow.

The NPV of the strategy was the sum from time period 0 through $\mathrm{T}$, in which the final time period, $\mathrm{T}$, was determined by a predetermined cutoff criterion, such as the maximum number of AI:

$$
\mathrm{NPV}=\sum_{\mathrm{t}=0}^{\mathrm{T}}\left[\frac{1}{(1+\mathrm{r})^{\mathrm{t}}} \times \mathrm{NCF}_{\mathrm{t}}\right],
$$

where $\mathrm{r}$ is the discount rate and $\mathrm{NCF}_{\mathrm{t}}$ is the net cash flow in period $t$.

Sensitivity analyses were performed for differing costs of labor, minutes required per injection, $\mathrm{CR}$, and labor efficiency of heat detection, holding all other variables constant. Further, sensitivity analysis was conducted for cows of different lactations. Heat detection labor efficiency was altered by holding the AI submission rate constant while changing labor hours required to achieve that performance. Efficiency in giving injections was altered by adjusting labor minutes required per single injection. Additionally, sensitivity to hourly labor costs was assessed to determine which types of programs had the greatest expected net present value, holding all other variables constant.

Baseline assumptions for $\mathrm{CR}$, costs per AI, time spent on heat detection per day, time required to give a single injection, and costs per injection for GnRH and $\mathrm{PGF}_{2 a}$ were obtained from the survey averages. The baseline values used were a CR of $38 \%$ (calculated by using average services per conception reported in the survey of 2.66 , as $1 /$ services per conception) and an $\mathrm{AI}$ submission rate of $100 \%$ under synchronization protocols (because cows are assumed bred by TAI). An AI submission rate of $65 \%$ was assumed under visual heat detection, although the labor hours necessary to achieve that submission rate were varied to assess sensitivity to labor efficiency. Cost per AI of $\$ 17.30$, time spent on heat detection of 2.15 labor $\mathrm{h} / \mathrm{d}$ (which, assuming a group size of 100 cows, would be $1.29 \mathrm{~min} /$ cow), 2.1 min required to give a single injection, and costs per injection of $\mathrm{GnRH}$ and $\mathrm{PGF}_{2 \alpha}$ of $\$ 3.59$ and $\$ 2.52$, respectively, were taken from reported survey averages.

\section{RESULTS AND DISCUSSION}

Survey findings are discussed with regard to how they inform the underlying economic analysis of factors affecting which farms use various reproductive management programs. Economic analyses, including sensitivity analyses of program costs to labor costs, visual heat detection efficiency, time required to give injections, and CR are presented to highlight those factors affecting on-farm decisions regarding reproductive program use.

The mean total herd size (including milking and dry cows) of survey respondents was 238 cows, with a range of 20 to 1,588 cows (57 respondents). Herds of fewer than 200 cows accounted for $69 \%$ of the respondents. In $2005,40.1 \%$ of operations in the US dairy industry had fewer than 200 milk cows (National Agricultural Statistics Service, 2007). Individual states varied considerably in farm size distributions, with New York, 
Michigan, and Wisconsin having had, respectively, $53.5,45.5$, and $68.5 \%$ of their farms with fewer than 200 cows (NASS, 2007).

To complete an economic assessment of the costs associated with various reproductive management strategies, an estimate of the associated labor costs was calculated. The average pay rate for hired managers was $\$ 12.78 / \mathrm{h}$. A range of values for the opportunity cost of labor from $\$ 6.00$ to $\$ 20.00 / \mathrm{h}$ were used to assess the sensitivity of the reproductive programs to on-farm labor costs, and to solve for break-even labor costs at which one program became preferred over another based on expected NPV criteria.

Overall, 78 and $64 \%$ of farms surveyed indicated that AI was used to breed cows and heifers, respectively, for at least some services. Zwald (2003), in comparing 14,500 herds, found that approximately half of the herds used a bull for at least some services. Caraviello et al. (2006), in their survey of dairy farms, also sought to determine the extent of AI use and found that 58 of the 103 herds surveyed, or 56\%, used solely AI. In the survey, the most frequent reason why AI was not solely used to breed cows was a lack of labor for heat detection and to perform AI, with 35\% of total responses (34 total responses for cows). Additionally, semen cost was cited in $24 \%$ of responses, "other reasons not listed" was cited in $20 \%$ of responses, using a cleanup bull was cited in $12 \%$ of responses, and lacking handling facilities was cited in $9 \%$ of responses.

Visual heat detection without the use of aids was the most prominent heat detection method used in both cows and heifers. The person responsible for heat detection will likely affect the true cost of a heat detection program because the owner or herdsman will likely have a greater labor cost than other farm employees. Of the 42 responses to this question, the person most commonly responsible for heat detection was the owner, with $55 \%$ of responses, followed by a shared responsibility by all employees, receiving $26 \%$ of responses; herdsman, with $17 \%$ of responses; and milkers, with $2 \%$ of responses.

If visual heat detection was being used in either cows or heifers, respondents were asked to provide additional information regarding how long animals were observed each time and who was responsible for heat detection. Of those farms reporting the use of visual heat detection in cows or heifers, on average, $78 \%$ of the cows and $90 \%$ of the heifers on those operations were bred solely by visual heat detection. The average overall AI submission rate reported for cows was $52 \%$. On average, cows and heifers were observed for estrus 3 and 2.2 times/d, respectively. These observation frequencies for heat detection were similar to those of Caraviello et al. (2006), who reported that cows were checked for estrus
2.8 times/d on weekdays and 2.5 times/d on weekends. In addition, Stevenson (2003) indicated in a survey of top dairy herds, as measured by yearly rolling herd averages, that cows were observed for estrus, on average, 3.1 times/d. Of the 31 farms reporting heat detection times, the average times spent observing cows and heifers were 43 and 19.5 min/observation, respectively. Previous survey results by Caraviello et al. (2006) indicated cows were observed for 27 min on weekdays and $25 \mathrm{~min}$ on weekends per observation. Compared with the report of Caraviello et al. (2006), survey respondents indicated that cows were observed for a longer time and heifers were observed for a shorter time. The total costs associated with visual heat detection per cow will be determined by the number of cows being observed or by the group size observed at a single time. Program costs per cow are sensitive to the number of cows being observed in a group at a single time.

Synchronization programs aim either to shorten the time during which cows or heifers must be observed or to eliminate the need for estrus detection entirely with TAI. Timed ovulation allows cows to be bred by appointment, thereby eliminating the need for heat detection, and has pregnancy outcomes similar to those obtained through AI performed after heat detection (Pursley et al., 1995). Separate responses regarding synchronization programs were invited for cows and heifers to allow for different management programs. In total, 56 and 45 responses were received for whether any synchronization program was used in 2005 for cows and heifers, respectively. Synchronization programs were used proportionately more in cows than in heifers, with $45 \%$ of responses indicating the use of some synchronization program in cows compared with only $27 \%$ in heifers. Respondents not using synchronization programs were asked to select reasons from a multiple-choice list. Possible reasons provided for cows or heifers included the expense of synchronization programs, manager or breeder preference to breed cows by visual heat detection, inadequate facilities to restrain cows for injections, lack of management time to manage a synchronization program, not being convinced of the benefits of synchronization, poor previous CR to TAI, and other. A summary of the responses for why synchronization programs were not used is provided in Table 1.

A list of common synchronization programs was provided, and respondents were asked to select any programs they used on cows or heifers or to provide information on any other protocols they used, and to explain what proportion of the herd each program was used on. Separate answers were encouraged for heifers and cows to allow for differing management programs. More responses were received for programs used than 
Table 1. Summary of survey results regarding reasons why synchronization programs were not used for cows or heifers ${ }^{1}$

\begin{tabular}{lrc}
\hline & \multicolumn{2}{c}{ Responses, \% } \\
\cline { 2 - 3 } Reason & Cows & Heifers \\
\hline Synchronization protocols too expensive & 16 & 12 \\
Prefer to breed to visually detected estrus & 28 & 24 \\
Inadequate handling facilities & 4 & 21 \\
Lack of management time & 8 & 9 \\
Not convinced of the benefits of synchronization & 16 & 12 \\
Poor previous conception rate to TAI & \\
Other $^{3}$ & 8 & 3 \\
\hline
\end{tabular}

${ }^{1}$ Number of responses $=25$ and 43 for cows and heifers, respectively.

${ }^{2}$ Farms having experienced poor conception rates in the past to timed AI (TAI).

${ }^{3}$ Other reasons were provided by farm managers by selecting "other" in the multiple choice question and providing a self-written reason why synchronization programs were not used. Reasons commonly mentioned by farm managers included being an organic dairy or simply preferring natural service.

for the number of farms that reported having used a synchronization program because several farms used more than one synchronization program. Given the small sample of farms that used synchronization programs for heifers in 2005, the proportion of the herd having used each program was not calculated because of insufficient numbers of farms using each program to make the proportion of the herd valuable. Table 2 provides a summary of the responses for cows and heifers related to synchronization programs.

Respondents were asked to provide cost information for any treatments used in cows or heifers. Costs per dose were collected, and these varied significantly across farms in the survey. For example, $\mathrm{PGF}_{2 \alpha}$ costs per dose ranged from $\$ 1.25$ to $\$ 6.00 /$ dose. Given the large variation in costs per dose reported, the costs of hormones available to a specific farm may indeed be different from those available to another, and such differences may alter decisions made regarding reproductive management programs. When farms were sorted into groups of 100 cows or fewer, 101 to 200 cows, and more than 200 cows, the costs for GnRH per dose were $\$ 4.49, \$ 3.13$, and $\$ 2.70$, whereas $\mathrm{PGF}_{2 \alpha}$ costs were $\$ 3.10, \$ 2.13$, and $\$ 2.10 /$ dose. Veterinarians reported to Nebel and Jobst (1998) hormone costs of $\mathrm{PGF}_{2 \alpha}$ at an average cost of $\$ 3.30 /$ dose, with a range of $\$ 2.50$ to $\$ 5.50 /$ dose, and of GnRH an average cost of $\$ 7.27 /$ dose, with a range of $\$ 4.50$ to $\$ 14.00 /$ dose.

Respondents were asked the amount of time needed per cow to give a single injection and the person responsible for giving synchronization program-related injections. The time required for injections and facilities used for injections varied considerably among farms, ranging from $17 \mathrm{~s}$, where shots were given to cows already in the milking parlor, to $10 \mathrm{~min}$, where heifers had to be sought out individually in a free stall and put into a headlock. Of the 26 farms responding to this question, the average time taken to give an injection was $2.1 \mathrm{~min}$. Twenty-seven responses were received regarding the person responsible for injections related to synchronization programs, with the person on the dairy responsible for synchronization-related injections the majority of the time being the owner, with $59 \%$ of responses. Following the owner in order of frequency were the herdsman or herd manager, the milker, the AI technician, and family members.

\section{Economic and Management Implications}

Prior reproductive performance was important in assessing which reproductive management program was the optimal program for a given farm. Farms that have

Table 2. Summary of survey responses regarding synchronization programs used ${ }^{1}$

\begin{tabular}{|c|c|c|c|}
\hline \multirow[b]{2}{*}{ Question } & \multicolumn{2}{|c|}{ Responses, \% } & \multirow{2}{*}{$\begin{array}{l}\text { Cows on which the } \\
\text { program was used, } \%\end{array}$} \\
\hline & Cows & Heifers & \\
\hline Ovsynch & 38 & 33 & 42 \\
\hline Presynch & 13 & 7 & 72 \\
\hline Cosynch & 6 & 0 & 57 \\
\hline Heatsynch & 2 & 0 & 10 \\
\hline $\mathrm{CIDR}^{2}$ with $\mathrm{PGF}_{2 a}$ & 19 & 20 & 7 \\
\hline Targeted breeding protocol & 6 & 20 & 63 \\
\hline Single $\mathrm{PGF}_{2 \alpha}$ injection (with $\mathrm{AI}$ on detected estrus) ${ }^{3}$ & 13 & 20 & \\
\hline Single $\mathrm{PGF}_{2 \alpha}$ injection with $\mathrm{TAI}^{4}$ & 2 & 0 & \\
\hline Other & 2 & 0 & 14 \\
\hline
\end{tabular}




\section{First-Lactation Cow NPV of Breeding Programs (Subject to Breeding Cutoff Criteria of $6 \mathrm{Al}$ )}

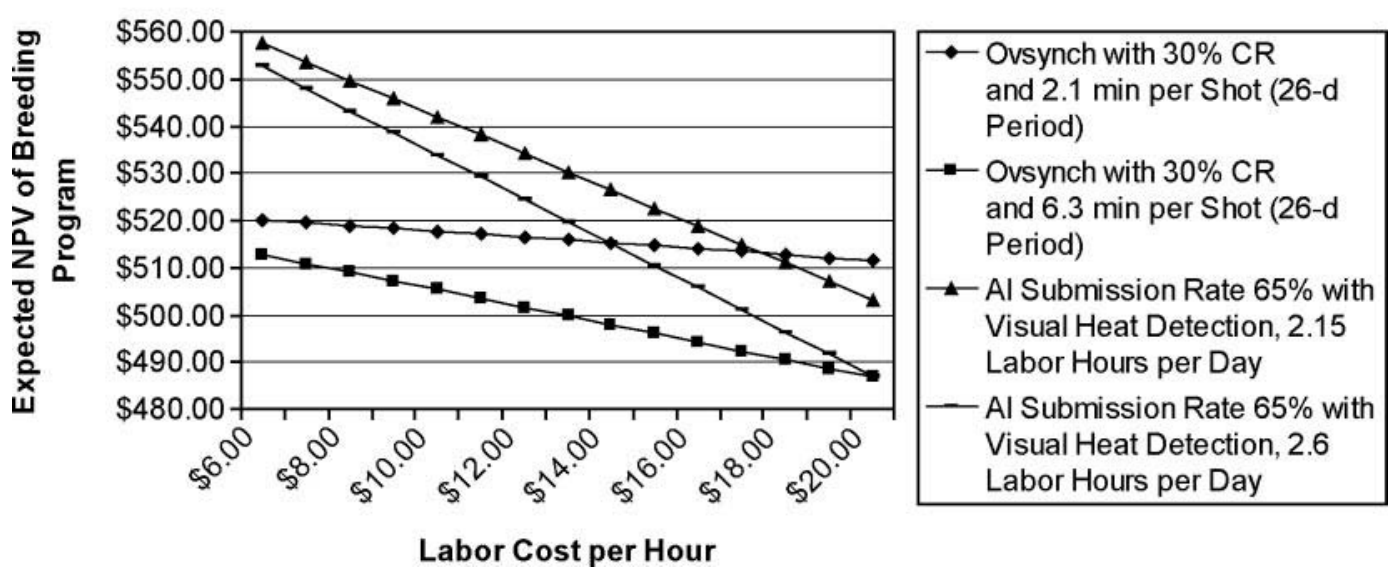

Figure 1. Net present values (NPV) of reproductive management programs for first-lactation cattle. $\mathrm{CR}=$ conception rate.

already experienced success in their reproductive management programs will have less economic incentive to pursue reproductive management changes. When individual farms are assessing a potential change in a reproductive management program, the prior level of performance is a key determinant. Farms that have experienced success with visual heat detection, as measured by high levels of efficiencies in detecting cows in heat, for example, are more likely to find that the expected NPV of the visual heat detection program remains greater than that of potential synchronization programs under more labor cost scenarios than farms that have not experienced such success. Beyond past levels of performance, managerial knowledge and experience with a given program affect their willingness to adopt programs. When looking specifically at synchronization programs, and in comparing such programs with visual heat detection programs, the resynchronization period is a key determinant of which program provides greater value. In short, the shorter the period of resynchronization, the greater the value of the program, and the lower the labor cost at which the synchronization program provides greater value than the visual heat detection program.

Farm human resource management and work environment factors must be taken into account when making decisions regarding reproductive management. Farm managers may prefer to work in a particular environment or to work with only family labor, rather than expanding the dairy and managing several employees. Human resource management challenges were identified by Caraviello et al. (2006) in their survey of dairy herd managers, in which managers identified finding

Journal of Dairy Science Vol. 91 No. 10, 2008 good employees as the greatest labor challenge, followed by training and supervising employees.

Focusing on reproductive programs using AI, we calculated the expected NPV for visual heat detection and synchronization protocols and performed sensitivity analyses. Figure 1 has the program costs for visual heat detection and Ovsynch for first-lactation animals, assuming a group size of 100, across labor costs ranging from $\$ 6.00$ to $\$ 20.00 / \mathrm{h}$. The cutoff criterion used for the programs in Figure 1 was that the cow was bred for 6 AI. Specifically, Figure 1 shows a comparison of the value of an $\mathrm{AI}$ submission rate of $65 \%$ achieved with 2.15 labor $\mathrm{h} / \mathrm{d}$ with the same program valued when using 2.6 labor $\mathrm{h} / \mathrm{d}$ for visual heat detection programs. The breeding periods assumed in Figures 1, 2, and 3 were a 21-d period for visual heat detection and a 26 -d period for synchronization. The difference between the expected NPV for the 2 visual heat detection programs highlights the difference that labor efficiency in heat detection makes in program selection. The scenario in which a $65 \% \mathrm{AI}$ submission rate is obtained in 2.15 labor $\mathrm{h} / \mathrm{d}$ exhibits greater levels of labor efficiency in detecting heats than one using 2.6 labor $\mathrm{h} / \mathrm{d}$ to attain the same $65 \%$ AI submission rate. Additionally, Ovsynch scenarios in which a $30 \% \mathrm{CR}$ is achieved with per-injection times of 2.1 or 6.3 min are provided for comparison. Similarly, time for injections affects the total labor costs associated with the program, although the same injections are administered whether it takes 2.1 or $6.3 \mathrm{~min} / \mathrm{shot}$.

Break-even values were calculated to determine the program with the greatest expected NPV, based on the on-farm labor costs, holding other parameters constant. In Figure 1, it is apparent that the visual heat detec- 


\section{Second-Lactation Cow NPV of Breeding Programs}

(Subject to Breeding Cutoff Criteria of 6 Al)

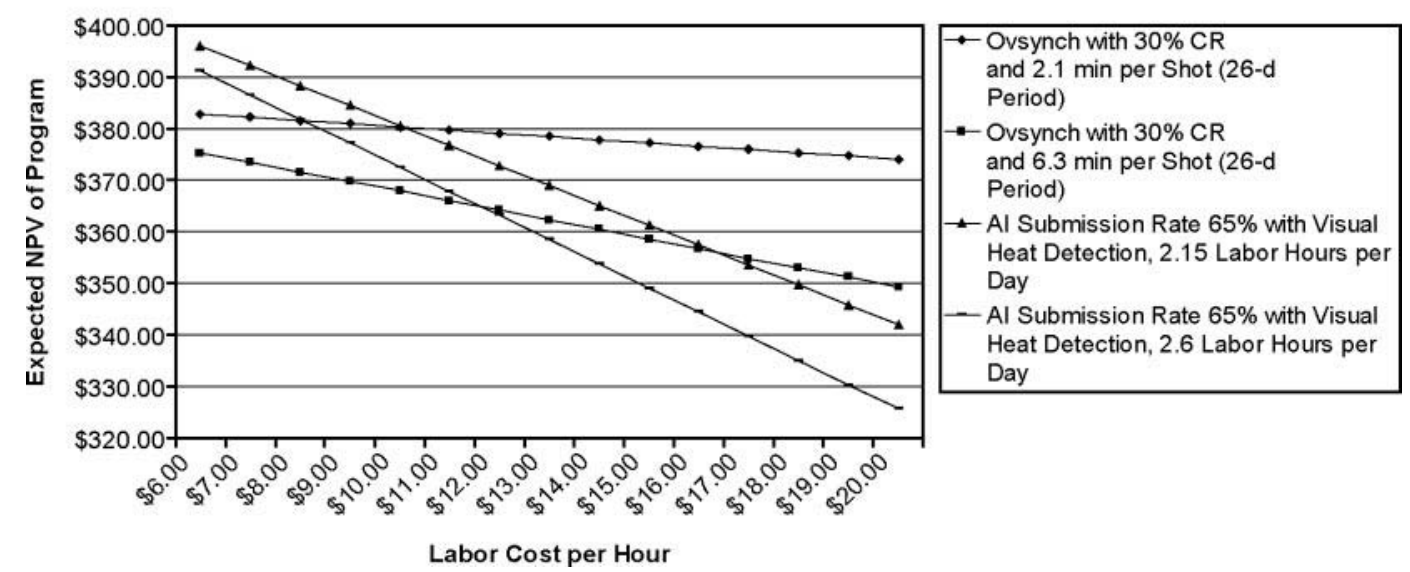

Figure 2. Net present values (NPV) of reproductive management programs for second-lactation cattle. CR $=$ conception rate.

tion programs, which use a great deal of labor, have higher NPV at low labor costs, whereas the Ovsynch program has higher NPV at high labor costs. When looking at the visual heat detection program, in which a $65 \%$ AI submission rate is achieved with 2.15 labor $\mathrm{h} / \mathrm{d}$, the visual heat detection program yields a greater NPV than Ovsynch with shots taking $2.1 \mathrm{~min}$, if labor costs are less than approximately $\$ 17.50 / \mathrm{h}$. If shots for Ovsynch take $6.3 \mathrm{~min}$, however, the visual heat detection program remains the better value program when labor costs are less than approximately $\$ 28.00 / \mathrm{h}$. When looking at the visual heat detection program in which an $\mathrm{AI}$ submission rate of $65 \%$ is achieved with 2.6 labor $\mathrm{h} / \mathrm{d}$, the labor costs at which Ovsynch becomes the better value program are lower than in the previous case, in which only 2.15 labor $\mathrm{h} / \mathrm{d}$ were used to achieve the same AI submission rate. In the case of 2.6 labor $\mathrm{h} / \mathrm{d}$ being used to obtain a $65 \% \mathrm{AI}$ submission rate, the visual heat detection program is the better value program when labor costs are less than approximately $\$ 14.00$ and $\$ 20.00 / \mathrm{h}$ for Ovsynch with shots taking 2.1 and $6.3 \mathrm{~min}$, respectively.

When second- and third-lactation cows were considered, the same basic principles held regarding sensitivities of programs to on-farm labor costs, although the decisions themselves, or at what labor costs a farm would select a given program, changed based on the expected NPV. The RPO of cattle, whether open or pregnant, are lower as the lactation number increases and expected productive life decreases; therefore, the values of the programs decrease in absolute value as the lactation number increases. Figure 2 has the ex-

Third-Lactation Cow NPV of Breeding Programs (Subject to Breeding Cutoff Criteria of 6 Al)

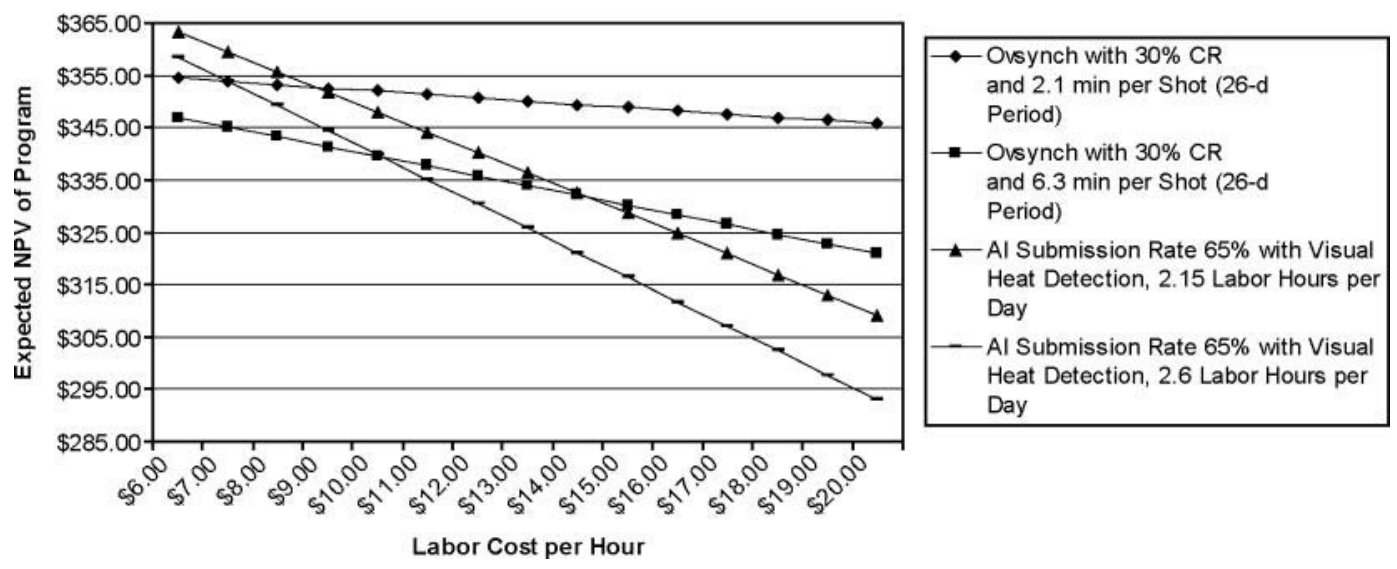

Figure 3. Net present values (NPV) of reproductive management programs for third-lactation cattle. CR = conception rate. 
Third-Lactation Cow NPV of Breeding Programs

(Subject to Breeding Cutoff Criteria of 6 Al)

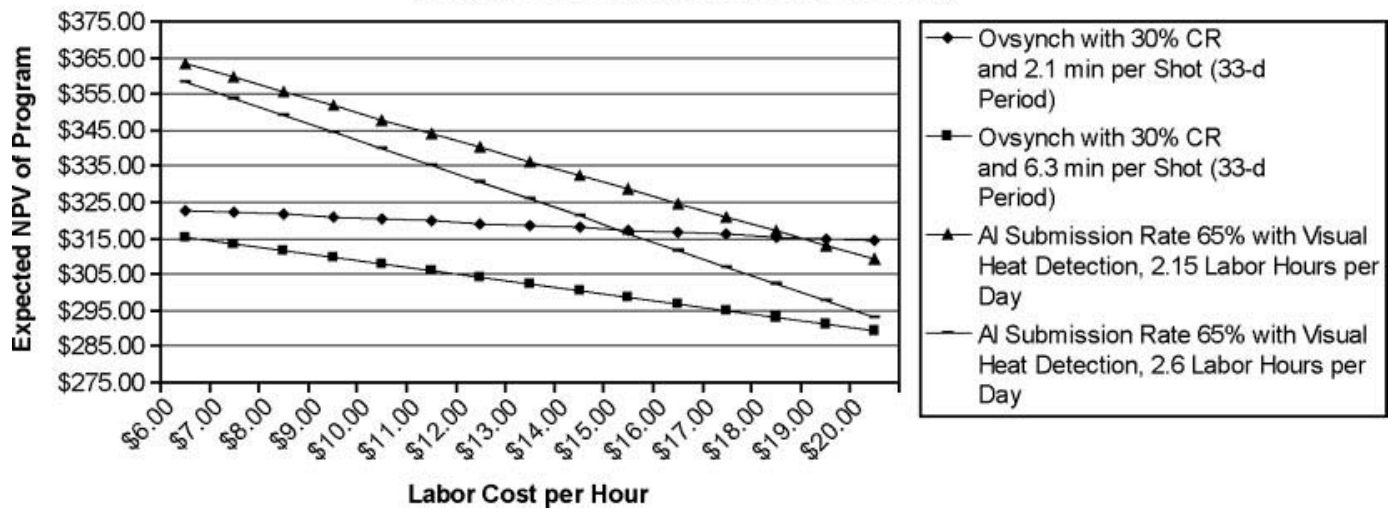

Figure 4. Sensitivity of net present values (NPV) of breeding programs to the synchronization breeding period for third-lactation cows. $\mathrm{CR}=$ conception rate.

pected NPV for various programs, subject to a 6-AI cutoff criterion for second-lactation cows. The labor costs above, in which the value of the Ovsynch program with 2.1 and $6.3 \mathrm{~min} / \mathrm{shot}$ is higher than in the visual heat detection program with a $65 \% \mathrm{AI}$ submission rate achieved in 2.15 labor $\mathrm{h} / \mathrm{d}$, are approximately $\$ 10.00$ and $\$ 16.00 / \mathrm{h}$, respectively. Alternatively, if it takes 2.6 labor $\mathrm{h} / \mathrm{d}$ to achieve the $65 \% \mathrm{AI}$ submission rate, the Ovsynch program with 2.1 and $6.3 \mathrm{~min} / \mathrm{shot}$ becomes the better value program for labor costs greater than approximately $\$ 8.00$ and $\$ 12.00 / \mathrm{h}$.

Expected NPV for various programs, subject to a 6 -AI cutoff criterion for third-lactation cows, are shown in Figure 3. With the 6-AI cutoff criterion, the labor cost above which the value of the Ovsynch program with $2.1 \mathrm{~min} / \mathrm{shot}$ is greater than the visual heat detection program with a $65 \%$ AI submission rate achieved in 2.15 labor h/d is approximately $\$ 9.00$. For the Ovsynch program with $6.3 \mathrm{~min} / \mathrm{shot}$ to have a greater value than the visual heat detection program in which a $65 \% \mathrm{AI}$ submission rate is achieved in 2.15 labor $\mathrm{h} / \mathrm{d}$, the labor cost per hour would need to be greater than approximately $\$ 14.00$. Alternatively, if it takes 2.6 labor $\mathrm{h} / \mathrm{d}$ to achieve the $65 \% \mathrm{AI}$ submission rate, the Ovsynch program with 2.1 and $6.3 \mathrm{~min} / \mathrm{shot}$ becomes the better value program for labor costs greater than approximately $\$ 7.00$ and $\$ 10.00 / \mathrm{h}$, respectively.

The synchronization program values are sensitive to the breeding period specified, or the number of days required to resynchronize cattle after a TAI. The breeding period specified is a key determinant of which program yields the greatest value under a given scenario. Figure 4 depicts the values of programs similarly to Figure 3, although the breeding period specified for the synchronization program is $33 \mathrm{~d}$, as opposed to $26 \mathrm{~d}$ as used previously. From Figure 4 it is clear that the lon- ger the resynchronization period, the greater the labor cost must be before synchronization is the most valued program. In Figure 4, the labor cost above which the value of the Ovsynch program with $2.1 \mathrm{~min} / \mathrm{shot}$ is greater than the visual heat detection program with a $65 \% \mathrm{AI}$ submission rate achieved in 2.15 labor $\mathrm{h} / \mathrm{d}$ is approximately $\$ 19.00$. For the Ovsynch program with $6.3 \mathrm{~min} / \mathrm{shot}$ to have a greater value than the visual heat detection program in which a $65 \% \mathrm{AI}$ submission rate is achieved in 2.15 labor $\mathrm{h} / \mathrm{d}$, the labor cost per hour would need to be greater than approximately $\$ 30.00$. Alternatively, if it takes 2.6 labor $\mathrm{h} / \mathrm{d}$ to achieve the $65 \% \mathrm{AI}$ submission rate, the Ovsynch program with 2.1 and $6.3 \mathrm{~min} / \mathrm{shot}$ becomes the better value program for labor costs greater than approximately $\$ 15.00$ and $\$ 21.00 / \mathrm{h}$, respectively.

Visual heat detection is more labor intensive, and thus more sensitive to increased labor costs, than a synchronization program. The relative sensitivities to labor costs of reproductive management programs can be seen when comparing the slopes of the lines representing values of heat detection versus synchronization programs in Figures 1, 2, and 3. As hourly labor costs increase, the expected NPV of the visual heat detection program decreases at a faster rate for visual heat detection programs than for the synchronization programs, illustrating the increased sensitivity of heat detection programs to labor costs compared with synchronization programs. Further, it can be seen in Figures 1, 2, and 3 that the better the efficiency in visual heat detection, the greater the labor cost must be before synchronization with Ovsynch has a greater expected NPV than visual heat detection. This supports the contention that current farm-level reproductive performance is important in assessing the highest value program for a particular farm. 
The labor efficiency in administering shots within a synchronization program or the labor efficiency with which heats are detected affect which program has the greatest NPV on a given farm operation. Further, the programs yielding the greatest NPV for cows in different lactations followed similar patterns, although the particular break-even labor costs among programs were different. The particular farm costs, labor efficiencies, lactation number, and reproductive performance level aid in determining which program yields the greatest NPV.

\section{CONCLUSIONS}

This analysis built on prior reproductive management studies and dairy industry surveys to inform the economic analyses of various reproductive management programs. Reproductive program costs were found to be highly sensitive to on-farm labor costs. Different types of reproductive management programs had different relative sensitivities to costs, such as on-farm labor costs. For example, visual heat detection requires more labor hours per cow and is therefore more sensitive to increasing labor costs than a synchronization program. As labor costs increased, when all other parameters were held constant, those reproductive management programs that are less sensitive to labor costs, namely synchronization programs, emerged as having greater expected NPV than visual heat detection programs. At what labor cost per hour synchronization emerged as having the greater expected NPV was dependent on farm-specific characteristics, such as on-farm costs, labor efficiencies, and the previous level of reproductive performance.

Current farm-level reproductive performance was found to be important in assessing the highest value program. The incentive for a farm to seek alternative programs for reproductive management decreased as farms had greater levels of current reproductive performance. Farms that have obtained high levels of visual heat detection efficiency, for example, have less incentive to adopt a synchronization program than those farms with less efficient visual heat detection.

Overall, reproductive management programs selected when seeking to maximize farm profitability through reproductive performance differ among farms because of varying on-farm costs, facilities, farm goals and values, previous levels of reproductive performance, and management styles. Through sensitivity to labor efficiencies in heat detection and administering injections, hourly on-farm labor costs, breeding periods, and program outcomes in $\mathrm{AI}$ submission rates and $\mathrm{CR}$, on-farm factors such as these have been highlighted as key determinants of the reproductive programs used on farms with given characteristics. By better understanding the factors that determine the programs used on farms, producers are better able to select programs that aid in effective and efficient reproductive management.

\section{REFERENCES}

Britt, J. H. 1985. Enhanced reproduction and its economic implications. J. Dairy Sci. 68:1585-1592.

Caraviello, D. Z., K. A. Wiegel, P. M. Fricke, M. C. Wiltbank, M. J. Florent, N. B. Cook, K. V. Nordlund, N. R. Zwald, and C. L. Rawson. 2006. Survey of management practices on reproductive performance of dairy cattle on large US commercial farms. J. Dairy Sci. 89:4723-4735.

Cassell, B. 2001. Service sires for problem breeders. Virginia Cooperative Extension Dairy Pipeline. http://www.ext.vt.edu/ news/periodicals/dairy/2001-07/servicesires.html Accessed on March 25, 2008.

De Vries, A. 2006. Economic value of pregnancy in dairy cattle. J. Dairy Sci. 89:3876-3885.

Fricke, P. M., D. Z. Caraviello, K. A. Weigel, and M. K. Welle. 2003. Fertility of dairy cows after resynchronization of ovulation at three intervals following first timed insemination. J. Dairy Sci. 86:3941-3950.

Groenendaal, H., D. T. Galligan, and H. A. Mulder. 2004. An economic spreadsheet model to determine optimal breeding and replacement decisions for dairy cattle. J. Dairy Sci. 87:21462157.

Meadows, C., P. J. Rajala-Schultz, and G. S. Frazer. 2005. A spreadsheet-based model demonstrating the nonuniform economic effects of varying reproductive performance in Ohio dairy herds. J. Dairy Sci. 88:1244-1254.

National Agricultural Statistics Service. 2007. Quick Stats Agricultural Statistics Data Base: U.S. \& All States DataDairy. Milk Cows by Size Groups: Operations. http://www.nass. usda.gov/QuickStats/ Accessed June 15, 2006.

Nebel, R. L., and S. M. Jobst. 1998. Evaluation of systematic breeding programs for lactating dairy cows: A review. J. Dairy Sci. 81:1169-1174.

Pursley, J. R., M. O. Mee, and M. C. Wiltbank. 1995. Sychnronization of ovulation in dairy cows using $\mathrm{PGF}_{2 \alpha}$ and $\mathrm{GnRH}$. Theriogenology 44:915-923.

Stevenson, J. 2003. Why natural service is not the solution. Hoards Dairyman (Oct. 25):6.

Wolf, C., S. Hanson, E. Wittenberg, and S. Harsh. 2002. Discount rates for dairy farms. Staff Paper 02-08. Agricultural, Food, and Resource Economics, Michigan State University, East Lansing.

Zwald, N. 2003. Does the extra effort of AI pay off? Hoards Dairyman (Oct. 25):11. 